\title{
L'avocat dans la défense pénale : de l'obligation de dire la vérité à un droit de mentir
}

\author{
Flavien Morard *
}

L'avocat pénaliste, dont le rôle est de défendre les intérêts de son client, doit-il être obligé de dire l'entière vérité au juge ? Peut-il mentir ? Trouver une juste balance entre l'avocat dénonciateur et l'avocat indigne de confiance est à la fois essentiel et difficile. Cet article intervient dans un paysage juridique encore flou et tente d'y apporter de la clarté. Il soutient qu'une obligation de dire la vérité de l'avocat s'oppose au rôle qu'il joue au sein de l'ordre juridique. En outre, une telle obligation viderait les garanties procédurales du justiciable de leur substance. Il s'agit également d'examiner dans quelle mesure l'avocat dispose d'un droit de mentir et d'en étudier la compatibilité avec le système juridique suisse actuel.

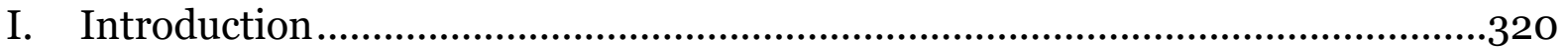

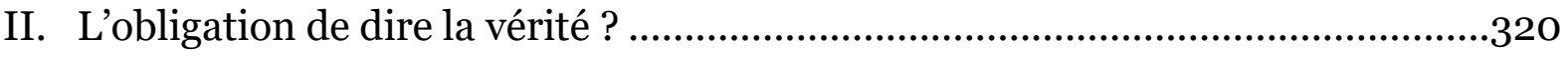

1. Les règles professionnelles de la LLCA ....................................................320

2. Le principe de la bonne foi .................................................................. 321

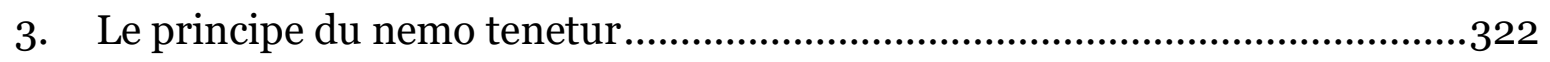

III. La nécessité d'une relativisation.......................................................................324

1. L'avocat en tant que garant de l'accès au droit .........................................324

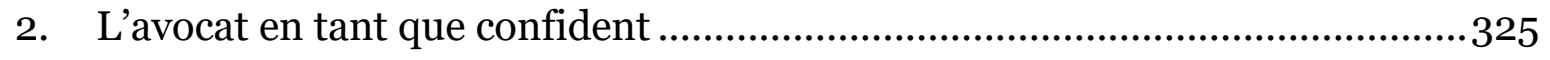

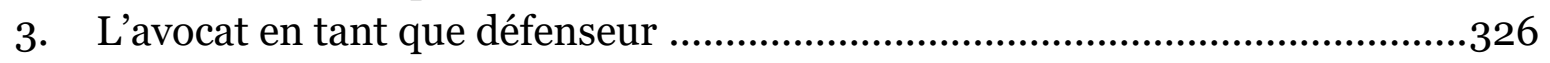

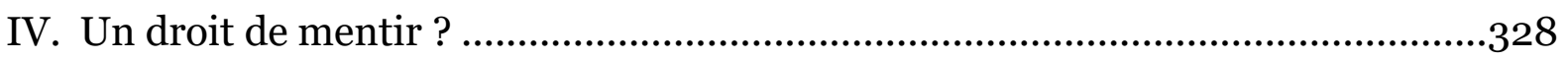

1. L'interdiction d'induire la justice en erreur .............................................328

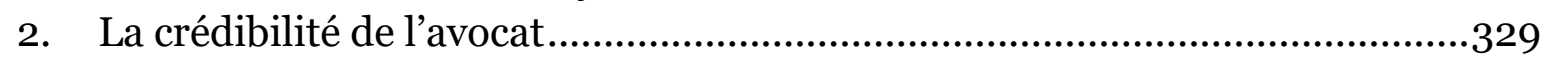

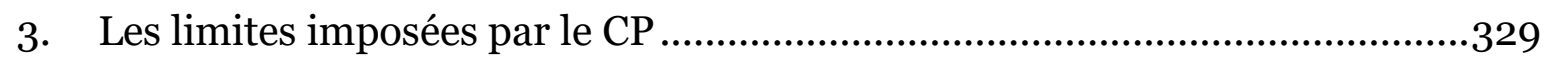

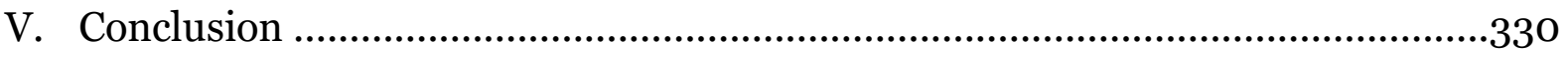

Zitiervorschlag: Flavien Morard, L’avocat dans la défense pénale : de l'obligation de dire la vérité à un droit de mentir, in: sui-generis 2017, S. 00

URL: $\quad$ sui-generis.ch/53

DOI: $\quad$ https://doi.org/10.21257/sg.53

* Flavien Morard, Mlaw, diplômé de l'Université de Zurich en été 2017. Il effectue actuellement un stage en préparation du brevet d'avocat. Cet article est une version raccourcie de son travail de master. L'auteur remercie le Prof. Marc Thommen pour sa précieuse collaboration. 


\section{Introduction}

1 La fonction de l'avocat jouit aujourd'hui d'une règlementation libérale plus ou moins forte selon la culture juridique du pays dans lequel il exerce ${ }^{1}$. Dans un État totalitaire, elle est limitée à celle d'un fonctionnaire poursuivant les intérêts de l'État avant ceux du justiciable. En revanche, au sein d'un ordre juridique purement libéral, l'avocat n'est soumis à aucune règle de droit public et le service juridique est entièrement laissé au marché libre ${ }^{2}$. En Suisse, l'avocat est mandaté par le client sur la base du droit privé, mais reste néanmoins soumis à des dispositions de droit public qui règlementent l'exercice de la profession3. Il doit obéir à deux intérêts parfois contradictoires : celui du justiciable qui désire être défendu de manière efficace et celui de l'État, qui veille à la bonne application du droit. Le TF dessine les contours de la profession d'avocat à l'ATF 106 Ia 100 par la formulation suivante :

«Der Anwalt ist 'Diener des Rechts' und 'Mitarbeiter der Rechtspflege' insoweit, als ihm die Aufgabe zukommt, die Rechtsuchenden bei der Verfolgung ihrer subjektiven Rechtsschutzinteressen zu beraten und zu unterstützen. Er nimmt damit eine Aufgabe wahr, ohne deren Erfüllung der Bürger seine Rechtsansprüche häufig nicht durchsetzen könnte und ohne deren Wahrnehmung die Verwirklichung der

\footnotetext{
Omlin Esther, Strafverteidigung - Grenzen der Wahrung von Parteiinteressen, Pratique Juridique Actuelle, Berne 2009, 74.

2 Schmid Niklaus, Handbuch des schweizerischen Strafprozessrechts, 2ème éd., Zurich/Saint-Gall 2013, no 1654 .

3 ATF 106 Ia 100, consid. 6b.
}

Rechtsordnung ganz allgemein in Frage gestellt wäre4. »

2 Selon cette approche, la fonction de l'avocat relève a priori davantage de la défense unilatérale des intérêts du mandant ainsi que du conseil et de l'assistance de ce dernier5. Le TF ne précise néanmoins pas si cette défense unilatérale doit être pondérée avec une obligation de dire la vérité ou non. Le présent article a pour but d'éclaircir cette incertitude.

3 Nous évoquons premièrement (II) les possibles fondements d'une obligation de dire la vérité de l'avocat. La deuxième partie (III) de cet article traite de la nécessité d'une relativisation de cette obligation à la lumière des fonctions clés de l'avocat : garant de l'accès au droit, confident et défenseur. Pour terminer nous poussons la réflexion plus loin en analysant la conformité d'un droit de mentir de l'avocat avec notre ordre juridique (IV).

\section{L'obligation de dire la vérité ?}

\section{Les règles professionnelles de la LLCA}

4 L'Assemblée fédérale vote en 2002 l'entrée en vigueur de la LLCA' 6 et soumet par la même occasion l'avocat à un catalogue de règles et d'obligations envers le justiciable ainsi qu'envers l'État7. Deux dispositions de la LLCA consistent en des

\footnotetext{
ATF 106 Ia 100, consid. 6b. ATF 106 Ia 100, consid. 6b.

LF du 23 juin 2000 sur la libre circulation des avocats (RS 935.61).

7 Message du 28 avril 1999 relatif à la loi fédérale sur la libre circulation des avocats (Message LLCA), FF 1999 5331, 5339 et 5367.
} 
restrictions du libre exercice de la profession de l'avocat. Il s'agit de l'obligation de soin et de diligence (sorgfältige und gewissenhafte Berufsausübung) de l'art. 12 lit. a LLCA, et de l'obligation de nature administrative ayant trait à l'obligation de s'inscrire au registre des avocats de l'art. 12 lit. j LLCA ${ }^{8}$. Cette dernière n'est pas traitée dans le présent article au vu de sa nature. Le devoir de soin et de diligence de l'art. 12 lit. a LLCA "permet d'exiger de l'avocat qu'il se comporte correctement dans l'exercice de sa profession 9 ", non seulement à l'égard de son client, mais également envers les autorités. Il doit s'abstenir de toute action qui pourrait porter atteinte à sa considération et à la confiance dont il devrait jouir ${ }^{10}$.

5 Cette disposition a une portée très générale qui ne permet pas d'établir clairement les obligations qui en découlent dans la pratique. Il convient de noter qu'une obligation de dire la vérité ne se trouve pas explicitement dans le catalogue des règles de la LLCA. À défaut, se pose la question de son fondement sur la base de l'obligation de soin et de diligence de l'art. 12 lit. a LLCA ${ }^{11}$ ou sur la base du principe de la bonne foi dont il est question ci-dessous.

8 Schiller Kaspar, Schweizerisches Anwaltsrecht Grundlagen und Kernbereich, Zurich 2009, $\mathrm{n}^{\circ} 226$ ss.

$9 \quad$ Message LLCA (n. 7), 5368.

10 Valticos Michel, art. 12, no 6 ss, in : Valticos Michel/Reiser Christian M./Chappuis Benoît (édit.), Commentaire romand, Loi fédérale sur la libre circulation des avocats (CoRo-LLCA), Bâle 2010.

11 Naegeli Georg, Darf man im Prozess lügen?, Revue de l'avocat 2010, 293.

\section{Le principe de la bonne foi}

6 Le principe de la bonne foi est ancré à l'art. 5 al. 3 Cst. ${ }^{12}$ ainsi qu'à l'art. 3 al. 2 lit. $1 \mathrm{CPP}^{13}$. L'art. 9 Cst. concrétise l'énoncé de l'art. 3 al. 2 lit. a CPP et en fait un droit constitutionnel subjectif garantissant un comportement non arbitraire de l'État envers l'individu : «toute personne a le droit d'être traitée par les organes de l'État sans arbitraire et conformément aux règles de la bonne foi ${ }^{14}$. " Le principe de la bonne foi s'adresse donc en premier lieu aux autorités et protège la confiance que le particulier fonde en elles ${ }^{15}$.

$7 \quad$ La doctrine n'est cependant pas unanime quant aux obligations que crée le principe de la bonne foi pour les particuliers. Certains auteurs affirment que ce principe limite le particulier à un usage de ses droits procéduraux sans abus de droit ${ }^{16}$. D'autres prétendent que l'obligation d'agir selon les règles de la bonne foi interdit au prévenu et à son avocat d'influencer de manière négative le cours de la justice et leur impose a contrario la tâche de participer à la bonne application

$12 \overline{\text { Constitution fédérale de la Confédération suisse }}$ du 18 avril 1999 (RS 101).

13 Code de procédure pénale suisse du 5 octobre 2007 (RS 312.0).

14 Thommen Marc, art. 3, no 46, in : Niggli Marcel Alexander/Heer Marianne/ Wiprächtiger Hans (édit.), Basler Kommentar, Schweizerische Strafprozessordnung: Jugendstrafprozessordnung I

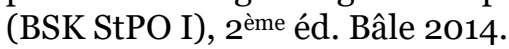

15 Mahon Pascal, art. 5, no 15 ss, in : Aubert JeanFrançois/Mahon Pascal (édit), Petit commentaire de la Constitution fédérale de la Confédération suisse du 18 avril 1999 (petit commentaire-Cst), Zurich/Bâle/Genève 2003; ATF 110 Ib 332, consid. 3.1.

16 ATF 127 I 34, consid. 2 a. 
du droit ainsi qu'à une obligation de dire la vérité17.

8 À la lettre l'art. 5 al. 3 Cst., les obligations découlant du principe de la bonne foi s'adressent à tout l'ordre juridique sans exception: «les organes de l'État et les particuliers doivent agir de manière conforme aux règles de la bonne foi. » L'art. 3 al. 2 lit. a CPP en est la concrétisation en matière pénale et limite l'application du principe de la bonne foi aux autorités pénales : "les autorités pénales respectent la dignité des personnes impliquées dans la procédure à tous les stades de celle-ci, elle se conforme notamment au principe de la bonne foi ${ }^{18}$.»

9 Le $\mathrm{TF}$, quant à lui, affirme que "le principe de la bonne foi ancré à l'art. $2 \mathrm{CC}^{19}$ est étendu (...) à l'ensemble des domaines du droit et en particulier à la procédure civile et pénale ${ }^{20}$. "Si ce raisonnement est justifié en procédure civile, il ne saurait s'appliquer sans autre en procédure pénale. Les deux procédures se différencient principalement en cela que la procédure civile oppose deux parties privées sur le plan horizontal, tandis que la procédure pénale se distingue par un déséquilibre de pouvoir vertical entre l'État et les particuliers (infra III.1.) ${ }^{21}$. En procédure civile, la maxime des débats interdit au tribunal d'éclaircir l'état de fait et le rend tributaire des affirmations faites par les parties ${ }^{22}$. À cet égard, l'art. 160 al. 1

17 Hauser Robert/Schweri Erhard/Hartmann Karl, Schweizerisches Strafprozessrecht, Bâle 2015, $266, \mathrm{n}^{\circ} 1 \mathrm{ss}$.

18 BSK-StPO I/Thommen (n. 14), art. 3, no 63.

19 Code civil suisse du 10 décembre 1907 (RS 210).

20 Arrêt du TF 1B_321/2013 du 30.10.2013, consid. 2.1; ATF 107 Ia 206, consid. 3.a.

21 BSK-StPO I/Thommen (n. 14), art. 3, no 63.

22 Hirtz Bernd, Der Umgang mit der Wahrheit im Zivilprozess, oder : ist der Rechtsanwalt im Zivil- lit. a $\mathrm{CPC}^{23}$ oblige les parties à collaborer à l'administration des preuves ainsi qu'à faire des dépositions conformes à la vérité. La procédure pénale est en revanche régie par la maxime de l'instruction. Cette dernière attribue la maîtrise du procès au juge, qui joue un rôle actif dans la recherche des éléments de preuves ${ }^{24}$.

10 Le principe de bonne foi, et par extension l'obligation de dire la vérité, ne peut donc pas être appliqué de la même manière en procédure pénale et en procédure civile. Ainsi, l'art. 9 Cst. vise uniquement les autorités étatiques et ne saurait servir de fondement à une obligation de dire la vérité de l'avocat.

\section{Le principe du nemo tenetur}

11 Le nemo tenetur est le principe selon lequel le prévenu n'a pas l'obligation de s'auto-incriminer. La Suisse a signé le Pacte international relatif aux droits civils et politiques adopté par l'Organisation des Nations Unies qui établit le principe du nemo tenetur à l'art. 14 al. 3 lit. g. En droit national, ce principe est ancré à l'art. 113 al. 1 CPP.

12 Le droit de ne pas contribuer à sa propre incrimination doit être distingué du droit au silence ${ }^{25}$. Si le premier concerne le comportement du prévenu pendant toute la procédure, lui garantissant ainsi le respect de sa volonté quant à sa participation active au procès, le deuxième a une portée plus restreinte et n'a trait qu'à la communication verbale du prévenu

prozess zur Wahrheit verpflichtet, AnwBl 2006, 780.

23 Code de procédure civile du 19 décembre 2008 (RS 272).

24 Schiller (n. 8), no 1602.

25 Arrêt de la CEDH, Saunders c. U Royaume-Uni, du 17 décembre 1991. 
envers les autorités ${ }^{26}$. Dans la mesure où l'accusé fait usage de son droit au silence, le droit de ne pas contribuer à sa propre incrimination exige que ce silence ne soit pas interprété en sa défaveur sur le plan juridique, sans quoi le silence de l'accusé l'auto-incriminerait ${ }^{27}$. Les conséquences factuelles du silence de l'accusé sont néanmoins dominées par un réflexe social, selon lequel la personne qui n'a rien à cacher, n'aurait pas de raison de garder le silence ${ }^{28}$. Même si le concept du nemo tenetur date de plusieurs centaines d'années, le silence de l'accusé reste inconsciemment considéré comme indice de sa culpabilité en pratique29. Par conséquent, une stratégie de défense doit être mise ne place au plus vite afin qu'elle soit cohérente et apte à déjouer les idées préconçues liées au silence du prévenu. Aussi, l'accusé doit pouvoir recourir au mensonge de manière à parer aux réflexes sociaux néfastes confondant parfois le silence avec un aveu tacite30.

Le prévenu désirant bénéficier de la protection conférée par le principe du nemo tenetur en particulier ne devrait pas en être empêché par la seule attribution d'un avocat, qui serait lui soumis à une obligation de dire l'entière vérité, découlant de l'obligation de soin et diligence de l'art. 12 al. 1 lit. a LLCA. Si les droits de l'avocat ne se calquent pas sur ceux du

$26 \overline{\text { Lieber Viktor, art. 113, } \mathrm{n}^{\mathrm{o}} \text { 1, in : Donatsch Andre- }}$ as/Hansjakob Thomas/Lieber Viktor (édit.), Kommentar zur schweizerischen Strafprozessordnung (StPO) (Commentaire CPP), Zurich/Bâle/Genève 2014 ; ATF 116 Ia 162, consid. 2d/aa.

27 ATF 131 IV 36, consid. 3.1; ATF 130 I 126, consid. 2.1.

28 Sommer Ulrich, Effektive Strafverteidigung, $3^{\text {ème }}$ éd., Munich 2016, 469, $\mathrm{n}^{\circ} 1128$ ss.

29 Sommer (n. 28), n ${ }^{\circ} 1131$ ss.

30 ATF 116 Ia 162, consid. 2d/aa ; ATF 109 Ia 160, consid. $4 \mathrm{~b}$. prévenu, ce dernier est amputé de ses garanties procédurales du simple fait d'être représenté en justice, ce qui ne saurait être l'intention du législateur31.

14 L'extension du droit au silence à l'avocat se justifie également du fait de l'interprétation conforme de la loi régie par l'art. 190 Cst32. Bien que le principe du nemo tenetur ne soit pas explicitement ancré dans la Cst., il découle du droit à un procès équitable qui est ancré aux art. $6 \mathrm{CEDH}$ et 29 ss Cst. L'art. 12 lit. a LLCA doit alors être interprété conformément au principe du nemo tenetur, de manière à ne pas imposer des obligations à l'avocat qui pourraient vider cette garantie de sa substance33. La question de savoir si en plus du droit de garder le silence, le droit de mentir du prévenu doit être étendu à l'avocat est traitée dans la troisième partie de ce travail (infra IV.).

$15 \mathrm{Au}$ terme de cette première partie nous retenons que l'obligation de dire la vérité de l'avocat ne dispose pas de fondements légaux suffisants pour être contraignante. Premièrement, la clause de soin et de diligence de l'art. 12 al. 1 lit. a LLCA ne contient pas d'obligation explicite de dire la vérité et doit être interprétée conformément au principe du nemo tenetur, excluant ainsi une telle obligation. Secondement, nous constatons que le principe de la bonne foi n'impose une obligation de participer à la bonne application du

${ }_{31} \overline{\text { Schiller (n. 8), no 246; BSK-StPO I/Ruckstuhl, }}$ art. $128, \mathrm{n}^{\circ} 1$.

32 Petit commentaire-Cst/Mahon Pascal (n. 15), art. $190, \mathrm{n}^{\circ} 15$.

33 Obrecht Willy, Die Stellung des Rechtsanwaltes bei der Wahrheitsfindung im Prozess, thèse, $\mathrm{Zu}$ rich/Bâle/Genève 1982, 56 et 62. 
droit ou à la recherche de la vérité ni au prévenu, ni à son avocat 34 .

\section{La nécessité d'une relativisation}

16 L'obligation de dire la vérité de l'avocat doit être relativisée de manière à ce que l'avocat puisse remplir ses trois fonctions principales : celle de garant de l'accès au droit et celle de confident et pour finir, celle de défenseur.

\section{L'avocat en tant que garant de l'accès au droit}

Le modèle de poursuite retenu dans le cadre du CPP se singularise par la concentration des pouvoirs au sein du Ministère public, ainsi que par le renforcement des droits de défense du prévenu, qui sont désormais garantis dès le début de la première audition35. Malgré le renforcement de ces droits, la nécessité d'une défense efficace est aujourd'hui accentuée par le déplacement du centre de gravité du procès pénal vers la phase préliminaire de la procédure, dont le procureur est devenu en quelque sorte le seul maître, de par sa nouvelle fonction de juge d'instruction ${ }^{36}$. En application de la maxime d'instruction, la fonction ordinaire du procureur durant cette phase est de rechercher les faits pertinents pour la qualification de l'acte incriminé, conformément à l'art. 6 al. 1 CPP. En vertu des art. 6 al. 2 CPP et 10 al. 1 CPP, il est également tenu de considérer les éléments à charge et à décharge du prévenu 37 . Il paraît toutefois, difficilement concevable

34 Schiller (n. 8), nos 1532 et 1532.

35 Piguet Christophe/Dyens Alexandre, Le Code de procédure pénale suisse a-t-il réellement renforcé les droits de la défense ?, in : Revue de l'avocat, vol. 18, Bâle 2015, $315 \mathrm{~s}$.

36 Piguet/Dyens (n. 35), 313 ss.

37 Piguet/Dyens (n. 35), 314. que le procureur puisse jouer le rôle d'autorité indépendante durant la phase préliminaire, s'il se voit attribuer le rôle de l'accusateur public par la suite ${ }^{8}$. Afin de parer à ce déséquilibre, le législateur formule à l'art. $130 \mathrm{CPP}$ une liste de situations, dans lesquelles l'accusé doit se faire assister par un avocat durant la procédure. Cette contrainte résulte d'une part d'une volonté d'assistance de l'État et sert d'autre part de contrepoids face au procureur, dont la compétence d'instruction revient exclusivement durant la phase préliminaire.

18 Même en dehors du champ d'application de la défense obligatoire de l'art. 130 $\mathrm{CPP}$, l'accusé ne peut que très difficilement remplir la tâche de se défendre luimême39. En effet, la tendance actuelle est de réglementer chaque domaine de la vie quotidienne, tout en sanctionnant pénalement la violation de nouvelles règles édictées par le législateur. La naissance de nouvelles incriminations rend l'application du droit de plus en plus complexe et hors de portée du simple justiciable40. En considérant la complexité actuelle d'une procédure pénale, le droit du justiciable d'être entendu ne peut être réalisé que difficilement sans l'assistance d'une personne possédant une formation juridique ${ }^{41}$.

19 Nous constatons à ce stade que l'accès au droit dépend aujourd'hui de facto de la défense par un avocat. Le justiciable dépend de son avocat afin de parer à la complexité d'une procédure pénale ainsi

38 BSK-StPO I/Ruckstuhl, art. 130, $\mathrm{n}^{0} 1$.

39 Dahs Hans, Handbuch des Strafverteidigers, 8 ème éd., Bonn 2015, 8 n 3 ss.

40 Rude-Antoine Edwige, L'éthique de l'avocat pénaliste, Paris 2014, 27.

41 Dahs (n. 39), 8, no 3 ss. 
qu'à la concentration du pouvoir en faveur de l'État.

\section{L'avocat en tant que confident}

20 La relation de confiance entre le prévenu et son avocat est indispensable afin que les intérêts du client puissent être sauvegardés de manière appropriée ${ }^{42}$. Il est nécessaire que le justiciable puisse se confier sans gêne ni méfiance et révéler à son mandataire tout ce qui est important à l'analyse de son dossier, sans avoir à craindre que l'avocat soit contraint de dévoiler ses confessions 43 . Le TF a également admis à plusieurs reprises «qu'il serait difficile à l'avocat, non complétement renseigné sur tous les faits importants, de conseiller judicieusement son client et de le représenter efficacement dans une procédure44. " Dans ce contexte, les informations désagréables et sensibles qui pourraient nuire au client sont particulièrement visées pour que la stratégie de défense puisse être établie en conséquence. En effet, l'avocat pourrait malencontreusement attirer l'attention du tribunal sur une partie de l'état de fait en défaveur de son client s'il n'en a pas été préalablement informé45.

21 Au vu de son importance considérable, le lien de confiance entre l'avocat et le justiciable doit être protégé. Une obligation de dire la vérité de l'avocat menace ce lien de plein fouet et mène à une situa-

42 CoRo-LLCA/Valticos (n. 10), art. 12, $\mathrm{n}^{\circ} 9$.

43 Bohnet François/Martenet Vincent, Droit de la profession d'avocat, Berne 2009, no 16 ; Bollmann Hans, Es kommt darauf an! Bemerkungen zu Anwaltsunternehmen und zu dem, was Anwälte so alles unternehmen, Berne 2013, 126 et 127.

44 ATF 117 Ia 341, consid. 4 ; ATF 102 Ia 516, consid. 3b ; ATF 112 Ib 606, consid. 1b.

45 Lubet Steven, Nothing but the truth, Why trial lawyers don't, can't and shouldn't have to tell the whole truth, New York 2002, 11. tion insatisfaisante : l'avocat pourrait favoriser l'ignorance sur la question de la culpabilité de manière à contrecarrer l'effet néfaste de l'obligation de dire la vérité, à savoir la perte de confiance de son client 46 . Il est toutefois problématique que l'avocat ne soit pas complètement renseigné sur les faits, et ne soit par conséquent plus à même de représenter efficacement les intérêts de son client en justice47.

22 Nous considérons que l'assistance d'un avocat en procédure pénale n'est pas un luxe de l'État de droit mais bel et bien une nécessité48. Une obligation de l'avocat de dire la vérité fait de la représentation en justice un cadeau empoisonné qui détériore la situation du mandant au lieu de l'améliorer ${ }^{49}$. Le justiciable a non seulement besoin d'une défense étant donné le déséquilibre des pouvoirs entre le justiciable et les autorités mais aussi au vu de la complexité qui se rattache à une procédure pénale. Il a également besoin d'un défenseur qui ne soit pas soumis à une obligation de dire la vérité, sans quoi la relation de confiance entre le justiciable et son avocat serait rompue.

23 Il s'agit à ce stade d'admettre la portée restreinte d'une obligation de dire la vérité en affirmant que l'avocat n'a pas le droit ni l'obligation de dire toute la vérité. En d'autres termes l'avocat a le droit de garder le silence sur les informations

46 Dahs (n. 39), 53, no 77.

47 ATF 117 Ia 341, consid. 4 ; ATF 102 Ia 516, consid. $3 \mathrm{~b}$.

48 Albrecht Peter, Die Funktion und Rechtstellung des Verteidigers im Strafverfahren, in : Niggli Marcel Alexander/Weissenberger Philippe (édit.), Strafverteidigung, vol. VII, Bâle 2002, 12, $\mathrm{n}^{\circ} 2.12$; Schiller (n. 8), $\mathrm{n}^{\circ} 78$.

49 Dahs (n. 39), 8, no 3. 
qui lui sont parvenues sur la base de sa relation de confiance avec son client50.

\section{L'avocat en tant que défenseur}

24 Nous souhaitons illustrer le rôle de défenseur de l'avocat à la lumière d'un cas pratique afin de mieux y exposer les enjeux des questions développées.

25 État de fait: Deux hommes, $A$ et $B$, tirent sur $C$ et le tuent. Les prévenus nient tout deux leur culpabilité et la procédure pénale qui s'ensuit ne parvient pas à établir les éléments de preuves nécessaires pour déterminer lequel des deux tirs a été mortel. $D$, avocat de $B$, plaide l'acquittement de son client devant le tribunal après que ce dernier lui a avoué sa culpabilité.

La réflexion qui est menée sur la base de cet état de fait a pour but d'étudier la conciliation de la défense de $B$ avec l'obligation de soin et de diligence de l'art. 12 al. 1 lit. a LLCA, la dénonciation calomnieuse de l'art 303 al. 1 CP51, la présomption d'innocence ainsi que le principe in dubio pro reo.

27 Tout d'abord, il s'agit d'analyser si dans le cas d'espèce le prévenu a le droit de nier sa culpabilité devant le juge, en vertu $\mathrm{du}$ principe du nemo tenetur sans se rendre coupable d'une dénonciation calomnieuse de l'art. 303 al. 1 CP. Dans l'affirmative, l'avocat devrait rester également impuni (supra II.3.). Si l'on arrive à la conclusion que le principe ne permet pas en l'espèce à l'accusé de nier sa cul-

$50 \overline{\text { Strafrechtsausschuss der Bundesrechtsanwalts- }}$ kammer, Thesen zur Strafverteidigung (thèse), vol.8, Munich 1992, thèse 19, 47.

${ }_{51}$ Code pénal suisse du 21 décembre 1937 (RS 311.0). pabilité, il s'agit d'analyser si l'avocat remplit lui-même les conditions de la dénonciation calomnieuse.

28 L'art. 303 al. 1 CP sanctionne celui qui aura dénoncé à l'autorité, comme auteur d'un crime ou d'un délit, une personne qu'il savait innocente, en vue de faire ouvrir contre elle une poursuite pénale ${ }^{2}$. La personne accusée ne doit pas être désignée explicitement; il suffit que cette dernière puisse être déterminée de manière implicite en considération des circonstances53. En outre, la dénonciation calomnieuse doit avoir conduit à l'ouverture d'une poursuite pénale à l'encontre de la personne faussement accusée. Si une procédure est déjà ouverte à l'encontre de la personne en question pour le même état de fait que celui sur lequel porte la fausse accusation, les affirmations calomnieuses ne sont pas sanctionnées par le CP54.

29 En niant sa propre culpabilité, B porte indirectement l'accusation contre A, qui est le seul à entrer en compte comme auteur au vu des circonstances. Il est alors sans autre possible de déterminer que la dénonciation calomnieuse vise $\mathrm{A}$, qui est poursuivi sur le plan pénal pour le même état de fait que B. Dès lors, si une poursuite pénale est déjà ouverte au moment de l'accusation, les affirmations calomnieuses de B ne remplissent pas les conditions de l'art. 303 al. $1 \mathrm{CP}$ et ne sont pas sanctionnées par le CP55. En l'espèce, une procédure pénale étant pendante à

52 Delnon Vera/Rüdy Berhard, art. 303, no 8., in : Niggli Marcel Alexander/Wiprächtiger Hans (édit.), Basler Kommentar, Strafrecht II (BSK StGB II), 3 ème éd., Bâle 2014.

53 BSK StGB II/Delnon/Rüdy (n. 52), art. 303, nº 9.

54 ATF 111 IV 159, consid. 2b.

55 ATF 111 IV 159, consid. 2b. 
l'encontre des deux hommes pour le même état de fait, les accusations que les prévenus peuvent faire l'un envers l'autre ne remplissent pas les conditions de l'art. 303 al. 1 CP. L'avocat doit par conséquent pouvoir plaider l'acquittement de B devant les tribunaux ${ }^{5}$.

30 En revanche, si B adresse ses allégations à l'encontre d'une tierce personne, envers laquelle aucune procédure pénale n'est ouverte, il n'est pas couvert par le principe du nemo tenetur car il peut être condamné au sens de l'art. 303 al. 1 CP57. Dans une telle situation, l'avocat peut quant à lui échapper à une sanction pénale uniquement s'il ne remplit pas luimême les conditions de cette disposition, par exemple s'il n'a pas la certitude de l'innocence de la personne faussement accusée ${ }^{5}$. Il faut alors distinguer la situation où l'avocat a connaissance certifiée des faits de celle où il n'en a que vaguement connaissance. Dans la mesure où le prévenu a avoué sa culpabilité à l'avocat, ce dernier se rend coupable de calomnie, car les conditions subjectives de l'infraction sont remplies. En revanche, si l'avocat n'a pas connaissance certifiée de la culpabilité de son client, il est critiquable qu'une grande partie de la doctrine, ainsi que la jurisprudence constante du $\mathrm{TF}$, partent du principe que l'intention éventuelle suffit pour remplir les conditions subjectives de l'art. 303 al. 1 CP59. La doctrine minoritaire est cependant d'avis que l'innocence de la per-

Dans ce sens : Willy (n. 33), 56, 62.

BSK StGB II/Delnon/Rüdy (n. 52), art. 303, nº 27.

BSK StGB II/Delnon/Rüdy (n. 52), art. 303, no 26.

59 ATF 80 IV 117, consid. D ; ATF 69 IV 80 ; ATF 72 IV 125 ; ATF 74 IV 47; ATF 76 IV 245 ; ATF 85 IV 80 ; Donatsch Andreas/Wohlers Wolfgang, Strafrecht IV, Delikte gegen die Allgemeinheit, $4^{\text {ème }}$ éd., Zurich 2011, 371; BSK StGB II/Delnon/Rüdy (n. 52), art. 303, nos 27 et 28. sonne faussement accusée doit être connue avec certitude par l'auteur de la dénonciation pour qu'il puisse être sanctionné6o.

31 L'extension terminologique jurisprudentielle à l'intention éventuelle est certes légitime s'agissant des difficultés qui peuvent se présenter au niveau des moyens de preuves, mais s'oppose à la défense efficace que le prévenu est en droit d'attendre de son avocat ${ }^{61}$. À cette fin, l'avocat doit pouvoir travailler sur la base d'hypothèses et d'éventualités, dans la mesure où elles sont nécessaires à sa prise de position ainsi qu'à la mise en place de sa stratégie de défense ${ }^{62}$. En outre, certains faits nécessaires aux fondements d'une prise de position ne sont parfois pas connus exactement par le mandant, dans cette mesure l'avocat n'a pas d'autre choix que de se fonder sur des éventualités 63.

32 De plus, étendre la terminologie de l'art. 303 al. $1 \mathrm{CP}$ à l'intention éventuelle va à l'encontre de la présomption d'innocence. Celle-ci permet précisément à l'avocat de plaider à décharge de son propre client sans pour autant plaider à charge d'autrui tout en se fondant sur des faits approximatifs et des hypothèses 64 . En d'autres termes, l'innocence de B ne doit pas forcément mener à la culpabilité d'une tierce personne. En l'espèce le juge devrait, selon notre appréciation, pren-

6o Petrzilka Werner, Zürcher Erläuterungen zum Schweizerischen Strafgesetzbuch, vol. II, Zurich 1942, 420.

61 BSK StGB II/Delnon/Rüdy (n. 52), art. 303, no 27.

62 Hafter Peter, Strategie und Technik des Zivilprozessrechts, Einführung in die Kunst des Prozessierens, 2 ème éd., Zurich/Bâle/Genève 2011, $\mathrm{n}^{\circ} 3200$.

63 Hafter (n. 62), 218, n. 1231.

64 BSK-StPO I/Riedo/Fiolka, art. 6, nº 63. 
dre en compte le principe in dubio pro reo. Si la preuve de la culpabilité ne peut pas être établie, ni à l'encontre de B, ni à l'encontre de $\mathrm{A}$, ce principe impose au tribunal de retenir l'état de fait dont la sanction est la plus favorable à l'encontre des deux prévenus, à savoir la tentative de meurtre de l'art. $111 \mathrm{CP}$ en lien avec l'art. 22 CP65.

Nous constatons que la défense de B n'est efficace qu'à condition que son avocat ait le droit d'émettre des hypothèses et de travailler sur des éventualités. En outre, il ne peut plaider l'acquittement de son client qu'en accusant A d'avoir tiré la balle meurtrière, toute en ayant connaissance certifiée de la culpabilité de son client. Ce mensonge est en l'espèce compatible avec le CP mais doit-on pour autant admettre un droit de mentir de l'avocat?

\section{Un droit de mentir ?}

34 Nous avons démontré jusque-là que l'obligation de dire la vérité de l'avocat doit être relativisée. Ce chapitre pousse la réflexion et analyse la compatibilité d'un éventuel droit de mentir de l'avocat avec notre ordre juridique. Nous traitons tout d'abord de l'interdiction d'induire la justice en erreur, puis des conséquences du mensonge sur la crédibilité de l'avocat. Les limites de ce droit imposées par le CP sont analysées en fin de chapitre.

65 Wohlers Wolfgang, Strafverteidigung vor den Schranken der Strafgerichtsbarkeit, StV 2001, art. 10, $\mathrm{n}^{\circ} 11$.

\section{L'interdiction d'induire la justice en erreur}

35 La doctrine majoritaire ainsi que la jurisprudence fédérale soutiennent une interdiction de l'avocat de perturber la recherche de la vérité en induisant les autorités en erreur ${ }^{66}$. Une telle interdiction n'est cependant pas ancrée explicitement dans une loi et le mensonge n'est pas interdit en tant que tel dans le CP (infra IV.III.).

36 Pour remédier à ce manque de base légale, l'interdiction de mentir doit être interprétée soit sur la base de l'art. 12 al. 1 lit. a LLCA ou du principe de la bonne foi de l'art. 5 Cst.67. Concernant le principe de la bonne foi, nous avons établi qu'il ne s'applique pas envers les particuliers dans le cadre de la procédure pénale (supra II.3.). La clause générale de l'art. 12 al. 1 lit. a LLCA ne saurait quant à elle en aucune façon transformer l'avocat en organe de l'État et le contraindre à ne dire que la vérité68. Cette disposition s'interprète à la lumière du principe du nemo tenetur et se limite à des situations telles que l'utilisation de preuves illégales, l'altération de pièces de la procédure, ou encore la falsification de documents ainsi que tout autre comportement sanctionné par le $\mathrm{CP}^{69}$.

37 En outre, l'existence d'une telle interdiction aurait des conséquences déplorables dans la pratique. Admettons que l'avocat plaide l'acquittement d'un client dont il

66 Brunner Alexandre/Henn Matthias-Christoph/ Kriesi Kathrin, Anwaltsrecht, Zurich 2015, no 86 ss ; Chappuis Benoît, La profession d'avocat, to-

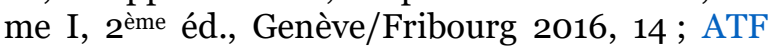
106 Ia 100, consid. 6b, ATF 133 I 259, consid. 3.4.

67 Schiller (n. 8), no 1512.

68 CoRo-LLCA/Valticos (n. 10), art. 12, no 34 .

69 CoRo-LLCA/Valticos (n. 10), art. 12, n ${ }^{\circ} 37$. 
connaît la culpabilité, il pourrait tenter de passer outre cette interdiction en reprenant indirectement les fausses affirmations de son client dans le procès. $\mathrm{Si}$ l'avocat prétend: Mon client, $A$, affirme ne pas avoir tué $B$, sa phrase ne peut en effet pas être considérée comme mensongère. Néanmoins, si l'avocat doit esquiver les questions du juge sur le plan rhétorique ou ne pas y répondre en gardant le silence pour protéger les intérêts de son mandant sans le dénoncer, il lui serait très difficile de cacher son intention. En effet, un juge expérimenté sait comment interpréter les réactions d'un avocat et comment déjouer ses feintes verbales. Il aurait de ce fait accès à des informations protégées par la relation privilégiée entre le prévenu et sa défense.

38 En résumé, une prétendue interdiction d'induire la justice en erreur n'a pas lieu d'être et s'efface faute de fondement légale devant le droit de mentir de l'avocat. Ce droit est nécessaire afin de garantir la défense efficace du justiciable d'une part mais aussi le respect de la relation de confiance entre l'avocat et son client, d'autre part.

\section{La crédibilité de l'avocat}

39 Une partie de la doctrine affirme qu'un droit de mentir de l'avocat pourrait affecter la crédibilité de la profession devant le juge. La plaidoirie de l'avocat pourrait être alors d'emblée soupçonnée d'être mensongère et les juges seraient alors contraints de l'étudier sous la perspective du mensonge ${ }^{70}$. Cela reviendrait à dire que la plaidoirie de l'avocat détermine la relation de confiance qu'il entretient avec

70 Bernhart Christof, Die professionellen Standards des Rechtsanwalts, Saint-Gall 2009, 115. le juge, et que cette confiance détermine quant à elle la crédibilité de l'avocat. Celle-ci se renforce à mesure des parutions des avocats devant le même tribunal 7 .

40 Cette relation de confiance entre le juge et l'avocat est néanmoins néfaste pour le justiciable, car elle pourrait encourager les avocats à accepter uniquement les mandats prometteurs sur le plan juridique de manière à augmenter leur capital confiance et de refuser ceux dans lesquels le droit n'est pas en faveur du client $^{72}$. Aussi, l'enjeu lié à la réputation et le capital de confiance que l'avocat entretient avec le juge est incompatible avec le rôle crucial que joue l'avocat en procédure pénale. En effet, il garantit l'accès à la justice et se place comme seul rempart face au pouvoir de l'État et celui du procureur en particulier (supra III.I)73. La relation de confiance que l'avocat entretient avec le juge ne saurait primer celle qu'il entretient avec son client, car c'est par cette représentation que l'avocat contribue à la bonne application du droit, et non par la volonté d'ajouter une victoire à un palmarès 74 . Nous sommes par conséquent d'avis que la crédibilité de l'avocat face au juge ne saurait être considérée comme un obstacle à un droit de mentir.

\section{Les limites imposées par le CP}

41 Une interdiction générale de mentir ne se retrouve pas explicitement dans la LLCA,

${ }_{71}$ Feldmann Adam, Who Wins in the Supreme Court? An Examination of Attorney and Law Firm Influence, Californie du sud 2015, 5 .

72 Muller Lukas, Dürfen Anwälte für ihre Mandanten lügen? Und falls ja, lohnt es sich?, Pratique Juridique Actuelle 2016, 552.

73 Schiller (n. 8), no 81.

74 Dahs (n. 39), $11 \mathrm{n}^{\circ} 7$. 
ni dans aucun autre texte de loi d'ailleurs. En effet, le CP suisse ne sanctionne pas le mensonge en tant que tel75. Peut-être parce qu'il est difficile voire impossible de déterminer et de prouver ce qui est mensonger, en particulier dans le cadre d'un procès où plusieurs réalités s'opposent et sont défendues. Même si le CP ne condamne pas le mensonge en tant que tel, certains aspects du mensonge sont contenus aux art. 173 à $177 \mathrm{CP}$, ainsi qu'à l'art. $303 \mathrm{CP}$ dont il était question plus haut (supra III.3.). La diffamation par exemple, sanctionnée par l'art. 173 ch. 2 $\mathrm{CP}$, condamne celui qui, en s'adressant à un tiers, aura faussement accusé une personne ou jeté sur elle un soupçon de tenir une conduite contraire à l'honneur. Des éléments du mensonge sont en outre contenus dans la disposition sur l'escroquerie à l'art. $146 \mathrm{CP}$. L'induction en erreur de l'art. $146 \mathrm{CP}$ a néanmoins un aspect patrimonial. Elle ne se limite pas à un comportement oral explicite, mais englobe également la manipulation d'objets et comportement de l'auteur pouvant induire en erreur ${ }^{76}$.

Le droit de mentir de l'avocat ne saurait servir à déjouer les dispositions du $\mathrm{CP}$ en la matière. Cependant, en dehors des cas réglementés sur le plan pénal, nous sommes d'avis qu'il doit appartenir à l'avocat de décider si, dans le cas d'espèce, il estime le mensonge nécessaire à la défense efficace de son client. Son appréciation doit se mesurer au degré d'inculpation et aux biens juridiques en jeu ainsi qu'à ses valeurs et considérations éthiques77. Envisager l'hypothèse d'un

75 Saliger Frank, Kann und soll das Recht die Lüge verbieten ?, in : Depenheuer Otto (édit.), Recht und Lüge, Munster 2005, 96.

76 Saliger (n. 75), 92 s.

77 Thèse 12 (n. 50), 38. droit de mentir de l'avocat n'est pas renoncer au système pénal actuel, qui peut s'en accommoder sans bouleversement.

\section{Conclusion}

43 L'application stricte de la jurisprudence du TF mentionnée en guise d'introduction concède à l'avocat un large rayon d'action pour mettre en place sa stratégie de défense ${ }^{78}$. Au terme de ce travail, nous pouvons résumer la portée de cette jurisprudence ainsi que les droits de défense de l'avocat qui s'y rattachent de la manière suivante.

44 "Der Anwalt ist "Diener des Rechts » und «Mitarbeiter der Rechtspflege» insoweit, als ihm die Aufgabe zukommt, die Rechtsuchenden bei der Verfolgung ihrer subjektiven Rechtsschutzinteressen zu beraten und zu unterstützen.79 »Par cette formulation, le TF établit expressément la primauté des intérêts privés du justiciable sur les intérêts publics de l'État. De ce fait, l'avocat doit pouvoir défendre son client devant les tribunaux et plaider son acquittement, même dans la mesure où il connaît sa culpabilité. Une relation de confiance est alors indispensable pour que l'avocat soit pleinement informé des faits et puisse soutenir et renseigner son client dans ses entreprises.

45 «Der Anwalt (...) nimmt damit eine Aufgabe wahr, ohne deren Erfüllung der Bürger seine Rechtsansprüche häufig nicht durchsetzen könnte ${ }^{80}$. » Outre sa fonction de défenseur des intérêts privés de son client, l'avocat est garant de

\footnotetext{
78 ATF 106 Ia 100, consid. 6b.

79 ATF 106 Ia 100, consid. 6b.

80 ATF 106 Ia 100, consid. 6b.
} 
l'accès au droit. Le justiciable est dépendant de ses services, sous peine de se retrouver désarmé face aux autorités de poursuite pénales auxquelles une augmentation crescendo de plusieurs réformes attribuent de plus en plus de compétences. En vertu de ce lien de dépendance, les garanties procédurales du justiciable, telle que le principe du nemo tenetur, doivent être étendues à l'avocat, sans quoi elles seraient vidées de leur substance.

Une obligation de dire la vérité absolue dans ce paysage juridique fait figure d'un éléphant dans un magasin de porcelaine. En effet, elle est inconciliable avec le rôle de l'avocat au sein de notre ordre juridique ainsi qu'avec les garanties procédurales du justiciable. L'avocat n'a par conséquent pas l'obligation de dire la vérité, mais le droit de garder le silence. Ce droit ne lui suffit néanmoins pas à mettre en place une défense efficace dans toutes les circonstances, comme celle où son client lui a avoué sa culpabilité, mais exige qu'il plaide son acquittement. En effet, une telle défense exige que l'avocat puisse nier la culpabilité de son client par des moyens actifs, sans quoi le juge n'aurait pas de peine à déjouer les effets de langage de l'avocat.

La frontière entre la représentation nécessaire des intérêts privés du justiciable et la complicité au crime est tracée par les dispositions du CP. Ces dispositions ont l'avantage, à la différence des règles de la LLCA, d'être rédigées avec plus de précision. Le mensonge n'étant pas condamné en tant que tel par le CP, l'avocat doit pouvoir s'en servir afin de défendre son client. Ce droit n'est toutefois pas absolu et ne doit pas servir à contourner les dispositions existantes en la matière. Si l'avocat doit avoir le droit de mentir sous ces conditions, il ne peut cependant pas être contraint à le faire. Sa décision doit être volontaire et reposer avant tout sur un jugement éthique qui soupèse les biens juridiques en question ${ }^{81}$.

48 «Der Anwalt (...) nimmt damit eine Aufgabe wahr ohne deren Wahrnehmung die Verwirklichung der Rechtsordnung ganz allgemein in Frage gestellt wäre ${ }^{82} »$. Pour finir, le TF reconnaît que de par son activité de défense unilatérale et de protection du justiciable face aux autorités de poursuite pénale, l'avocat sert l'État de droit au même titre que le procureur ou le juge. Tous visent le même idéal de justice avec des points de départ différents ${ }^{8}$. Telle est la fonction de l'avocat moderne et sa contribution à la bonne application du droit, celle dont Jacques Vergès esquisse les contours lorsqu'il fait l'éloge de l'avocat du futur, " capable de comprendre tous les hommes (...), faisant de ses procès une création permanente et d'une tragédie individuelle celle de tous, toujours en mouvement et assumant mieux que personne l'humanité tout entière ${ }^{84}$ ».
81 Rude-Antoine (n. 40), 185.

82 ATF 106 Ia 100, consid. 6b.

83 Dahs (n. 39), 11, no 7.

84 Vergès Jacques, Justice et littérature, Paris 2011, Sommaire. 